\title{
Recomendaciones para el tratamiento del asma durante la contingencia causada por COVID-19 en Colombia
}

\section{Recommendations for the treatment of asthma during the contingency caused by COVID-19 in Colombia}

\begin{abstract}
Audrey Piotrostanalzki, MD¹, Robín Rada, MD¹, Diana Rey, MD¹, Carlos Celis MD¹, IVÁn Solarte, MD¹,

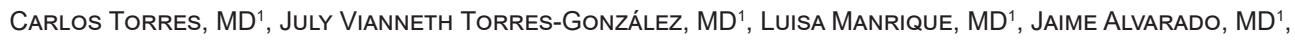

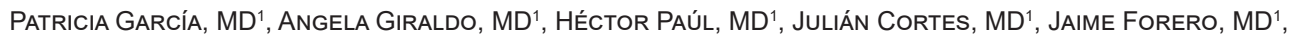

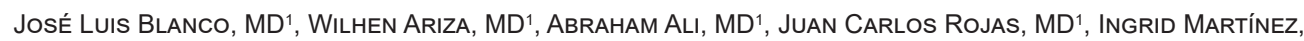
MD'1, Rafael Acero, MD¹, Jorge Quintero, MD¹, ERnesto Santiago, MD ${ }^{1}$, Isabel Palacios, MD' ${ }^{1}$, Lucila

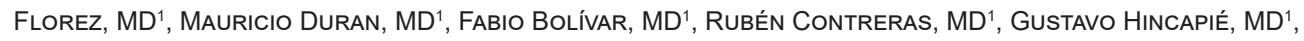

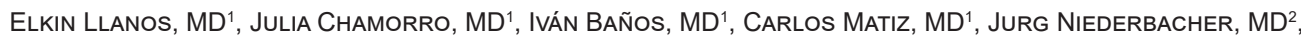
Edison Morales, MD³ ${ }^{3}$ Jorge Herman Piraquive, MD 4 .
\end{abstract}

\section{Introducción}

El asma es una enfermedad pulmonar inflamatoria crónica, que se caracteriza por episodios recurrentes de obstrucción bronquial, con una frecuencia y gravedad variable. En Colombia, su prevalencia es del 12\% (12 de cada 100 colombianos tienen la enfermedad). Para su control se emplean los esteroides inhalados solos o asociados con otros medicamentos.

El COVID-19 es la sigla de la enfermedad causada por el coronavirus SARS$\mathrm{CoV}-2$, que afecta varios órganos y sistemas, principalmente el sistema respiratorio, y se manifiesta generalmente por síntomas de infección respiratoria aguda (1).

Los coronavirus son una familia de virus que pueden transmitirse a los humanos por vía de animales intermediarios. De estos, se conocen 7 especies que generan la enfermedad en humanos, una de las cuales es el SARS-CoV-2, causante del nuevo COVID-19. De estas 7 especies, hay 3 de mayor importancia por la gravedad del compromiso causado: SARS-CoV, que fue causal de enfermedad en Guangdong, China, en los años 2002 y 2003; MERS-CoV, que generó enfermedad pulmonar grave de alta mortalidad en el año 2012; y, actualmente, el SARS-CoV-2, causante del COVID-19(1).

A principios de diciembre de 2019, en la ciudad de Wuhan, capital de la provincia de Hubei, China, se describieron los primeros casos de neumonía de origen desconocido. Luego, se logró identificar el patógeno, un nuevo betacoronavirus de ARN envuelto, denominado coronavirus 2 del síndrome respiratorio agudo severo (SARS-CoV-2) (2).

La Organización Mundial de la Salud (OMS) anunció que la enfermedad por coronavirus 2019 (COVID-19) es una emergencia de salud pública de preocupación internacional, la cual ha evolucionado rápidamente, siendo declarada pandemia, con un espectro amplio de manifestaciones y gravedad de sus síntomas; que clasifica su enfermedad como leve, grave (severa) y crítica (3).
${ }^{1}$ Especialistas en Medicina Interna y Neumología; Comité de Asma, Asociación Colombiana de Neumología y Cirugía de Tórax.

${ }^{2}$ Médico especialista en Pediatría y Neumología pediátrica, Presidente de la Asociación Colombiana de Neumología Pediátrica; Profesor titular Universidad Industrial de Santander; Bucaramanga, Colombia.

${ }^{3}$ Médico especialista en Alergología, Presidente, Asociación Colombiana de Alergia, Asma e Inmunología; Medellín, Colombia.

${ }^{4}$ Médico internista, fellow Neumología, Hospital Militar Central; Bogotá, Colombia.

Autor de correspondencia:

Dra. Diana Rey.

Correo electrónico:

dianarey85@gmail.com

Recibido: $17 / 05 / 2020$

Aceptado: 30/05/2020 
Hasta la fecha, la mayoría de los estudios sugiere que los pacientes con asma no tienen mayor riesgo de adquirir infección por COVID-19, al compararse con la población general $(4,5)$. No obstante, si el paciente con asma llega a contraer la infección por COVID-19, podría resultar en la pérdida del control de la enfermedad y crisis asmática, si asumimos que las infecciones del tracto respiratorio son una causa común de exacerbaciones de asma. (6). En este escenario es importante puntualizar estrategias de manejo para los pacientes asmáticos durante la pandemia COVID-19, que permitan mantener el máximo control de la enfermedad.

\section{Recomendaciones para el uso de esteroides inhalados}

Los pacientes con asma, tengan o no infección por COVID-19, deben continuar el uso de su medicación usual, siendo vital los esteroides inhalados, suministrados con inhaladores de polvo seco o inhaladores de dosis medida presurizados con inhalo-cámaras apropiadas (esto último, indispensable en niños) $(7,8)$.

La iniciativa mundial para el asma (GINA), 2020, recomienda que los pacientes con esta patología no deben suspender los medicamentos prescritos para el control de la enfermedad, siendo fundamental la continuidad o reinicio de los esteroides inhalados, ya que la suspensión o falta en el tratamiento podría conducir a un empeoramiento potencialmente peligroso del asma (9). Se recomienda que el paciente comunique siempre a su médico cualquier modificación del tratamiento o suspensión de aquellos que recibe para el control del asma (10). Respecto al uso de esteroides orales, que puedan requerirse durante el manejo de crisis asmática como ciclos cortos de tratamiento, deberán ser siempre evaluados por el médico tratante, según el contexto de cada paciente (11).

En caso de crisis asmática no se recomienda el uso de micronebulizaciones por razones de bioseguridad, ya que este procedimiento incrementa la generación de aerosoles y pueden diseminar la infección por COVID-19 (7). El uso de inhalador de dosis medida con el espaciador durante las crisis es adecuado, y su eficacia es similar. En casa, los espaciadores o inhalocamaras no deben compartirse $(11,12)$.

\section{Inmunizaciones}

Hasta el momento no está disponible una vacuna efectiva contra la enfermedad relacionada con el COVID-19, aunque los esfuerzos científicos están enfocados en su desarrollo (13). Sin embargo, no hay que olvidar que los pacientes con asma continúan con riesgo de infección por otros virus y bacterias previamente conocidos, como la influenza (virus causal de la gripa estacional) y el neumococo (principal causa de neumonía y otras infecciones respiratorias).

Las medidas de prevención, como el lavado e higiene de manos, continúan siendo las medidas más efectivas para evitar la infección $(14,15)$. Sin embargo, ante la disponibilidad de las vacunas de forma anual para la influenza y la vacuna del neumococo para los mayores de 60 años, los pacientes con asma deben continuar con la inmunización recomendada por el personal de la salud y las organizaciones internacionales, para disminuir el impacto de las infecciones respiratorias $(14,16,17)$.

\section{Recomendaciones de terapia biológica en pacientes con asma grave}

Se recomienda que los pacientes con asma grave continúen el tratamiento de terapia biológica durante la pandemia por COVID-19 $(18,19)$. Desde el punto de vista biológico se asume que la terapia biológica no afectaría negativamente la respuesta inmune a la infección viral (20).

Los pacientes podrán continuar la terapia biológica con la cual se encuentren en tratamiento: anti-IgE, anti-IL-5 y anticuerpos monoclonales anti-IL-4/IL-13. El beneficio se relaciona directamente con la reducción de exacerbaciones graves de asma y, por tanto, con la probabilidad de ingresar al sistema de salud durante el transcurso de la pandemia COVID-19.

En caso tal que se requiera iniciar terapia biológica en el paciente con asma grave durante la pandemia COVID-19, se debería considerar la aplicación en casa, usando los servicios de atención médica domiciliaria. Si esto no es posible, esta se puede iniciar en el centro de aplicación, preferiblemente con un máximo de 1-2 visitas, y luego considerar transición a la administración domiciliaria en la mayoría de los casos $(20,21)$. 


\section{Recomendaciones para los pacientes con asma durante la pandemia por COVID-19 (6, 22-24)}

- Tenga disponibilidad de su medicación de mantenimiento (crónica) por mínimo 1 mes de tratamiento.

- Evite la exposición a sustancias o alérgenos que aumenten sus síntomas de asma.

- Utilice, cuando sea posible, opciones de teleconsulta (telefónica o por internet) con su médico tratante para el control de la enfermedad.

- Solicite a su médico un plan de acción del asma en caso de que presente deterioro y requiera hacer ajustes por su propia cuenta para el control de su enfermedad.

- Cuando asista a lugares públicos guarde distancia de las personas que estén en el mismo lugar.

- Evite multitudes y contacto con personas enfermas.

- Evite viajes no esenciales.

- Manténgase en casa tanto como sea posible.

- Si convive en casa con alguien enfermo, mantenga su distancia y el aislamiento de la persona enferma en una habitación diferente dentro de su hogar, esto disminuye el riesgo de contagio.

- Evite compartir insumos personales, como vasos y toallas. Tenga precaución con el uso compartido y la limpieza de lapiceros, celulares, juguetes y utensilios que puedan estar contaminados con saliva, con especial control con aquellos de los niños (23).

- Mantenga su casa limpia y desinfectada, teniendo en cuenta la desinfección de superficies frecuentemente usadas, como el mesón de baño y la cocina, perillas de las puertas, interruptores, manijas, escritorios, teléfonos, teclados, baños, grifos y lavamanos. Evite el uso de aquellos desinfectantes que, en su caso, desencadenen ataques de asma.

- Siga las medidas de higiene, como el lavado de manos frecuente, y de aislamiento recomendadas por las autoridades locales durante la epidemia de coronavirus.
- Siga las recomendaciones de la autoridad local vigente respecto a pasos a seguir en caso de sospechar infección por coronavirus.

- No se deben realizar pruebas de función pulmonar, por ejemplo, espirometría, prueba de reactividad bronquial o medición de óxido nítrico exhalado, durante la epidemia del coronavirus.

- Tome las medidas que le permitan manejar el estrés en casa y estar con tranquilidad en medio de las dificultades. Recuerde que unos de los factores que pueden generar ataques de asma son el estrés y ansiedad, síntomas que pudieran presentarse durante la pandemia.

- Los niños y adolescentes, aunque en la mayoría de los casos sufren la enfermedad de forma leve, son altamente transmisores de la misma, incluso en su estado de asintomáticos portadores del virus; por tanto, se recomienda tenerlos alejados de los adultos mayores, mucho más si padecen de asma.

- Si cuenta con la posibilidad de atención domiciliaria, solicítela cuando haya indicaciones de hacerlo.

\section{Retos en el cuidado del paciente asmático durante la pandemia COVID-19 en Colombia}

Es necesario utilizar las tecnologías disponibles para mantener al paciente con enfermedades crónicas no transmisibles controlado y con medicación disponible para su tratamiento en casa, en este caso, el paciente con el diagnóstico de asma.

Como comunidad necesitamos adaptarnos rápidamente al cambio, en búsqueda de mantener nuestra población segura y saludable. El uso de telemedicina, por parte de profesionales de salud en casa, es una medida de contingencia orientada a la promoción de la salud y a la prevención de la enfermedad, puesto que permite mantener a la mayoría de los pacientes fuera de los hospitales y de servicios de consulta ambulatoria, apoya que, aquellos médicos que se encuentren en turnos de trabajo, tengan una menor exposición a la infección por coronavirus, y facilita que los pacientes no infectados permanezcan en menor riesgo de estarlo. 
La telemedicina se puede usar en el paciente con asma, reconociendo con responsabilidad hasta donde es posible valorar adecuadamente un paciente a distancia con el uso de la tecnología disponible, como cámaras, pantallas, micrófonos, parlantes y audífonos; lo cual es perfectamente válido para actos como registrar la evolución del paciente y la gravedad de la enfermedad, renovar la formulación de medicamentos controladores o aliviadores del asma, valorar la sospecha de comorbilidades, solicitar ayudas diagnósticas, dejando claro cuando se recomienda hacerlas en las actuales circunstancias de aislamiento social, prescribir un plan de acción de asma para el manejo de crisis, o indicar el escalado o desescalado de medicamentos controladores, cuando esto se requiera.

De todo lo actuado y decidido mediante la telemedicina deben quedar registros en la historia clínica del paciente, con la respectiva nota de que se hizo bajo esta modalidad, de común acuerdo con el paciente, e incluso el motivo para la misma; esto para los controles ulteriores y como soporte de las responsabilidades en lo actuado.

\section{Referencias}

1. Graham C, De la Cruz CS, Cao B, Pasnick S, Jamil S. COVID-19 disease due to SARS-CoV-2 (novel coronavirus). Am J Respir Crit Care Med. 2020;201:P7-8. doi: https://doi. org/10.1164/rccm.2014P7.

2. Huang C, Wang Y, Li X, Ren L, Zhao J, Hu Y, et al. Clinical features of patients infected with 2019 novel coronavirus in Wuhan, China. Lancet. 2020;395(10223):497-506. doi: https://doi.org/10.1016/S0140-6736(20)30183-5.

3. Guan W, Ni Z, Hu Yu, Liang W, Ou C, He J, et al. Clinical characteristics of coronavirus disease 2019 in China. N Engl J Med. 2020;382:1708-20. doi: 10.1056/NEJMoa2002032.

4. Halpin DMG, Faner R, Sibila O, Ramon Badia J, Agusti A. Do chronic respiratory diseases or their treatment affect the risk of SARS-CoV-2 infection? Lancet Respir Med. 2020;8(5):436-8. doi: 10.1016/S2213-2600(20)30167-3.

5. Epidemiology Working Group for NCIP Epidemic Response, Chinese Center for Disease Control and Prevention. The epidemiological characteristics of an outbreak of 2019 novel coronavirus diseases (COVID-19) in China]. Zhonghua Liu Xing Bing Xue Za Zhi. 2020;41(2):145-51. doi: 10.3760/cma .j.issn.0254-6450.2020.02.003.

6. Center for Disease Control and Prevention [Internet]. People with moderate to severe asthma. 2020. Última visita: 19 de marzo de 2020. Disponible en: https://www.cdc.gov/ coronavirus/2019-ncov/specific- groups/asthma.html
7. Rodriguez-Martinez CE, Sossa M, Lozano JM. Commercial versus home-made spacers in delivering bronchodilator therapy for acute therapy in children. Cochrane Database Syst Rev. 2008;(2):CD005536. doi: 10.1002/14651858.CD005536.pub2.

8. van Bever HP, Chng SY, Goh DY. Childhood severe acute respiratory syndrome, coronavirus infections and asthma. Pediatr Allergy Immunol. 2004;15(3):206-9. doi: 10.1111/j.13993038.2004.00137.x.

9. Global Initiative for Asthma [Internet]. Recommendations for inhaled asthma controller medications. 2020. Última visita: 19 de marzo de 2020. Disponible en: https://ginasthma.org/recommendations-for-inhaled-asthma- controller-medications/

10. Centre for Evidence-Based Medicine [Internet]. Inhaled steroids in asthma during the COVID-19 outbreak. Última visita: 19 de marzo de 2020. Disponible en: https://www.cebm. net/covid-19/inhaled-steroids-in-asthma-during-the-covid19-outbreak/

11. Ministerio de Sanidad, Consumo y Bienestar Social. Documentos técnicos para profesionales - coronavirus [Internet]. Última visita: 22 de marzo de 2020. Disponible en: https:// www.mscbs.gob.es/profesionales/saludPublica/ccayes/alerta$\mathrm{sActual} / \mathrm{nCov} /$ documentos.htm

12. Sociedad Española de Neumología y Cirugía Torácica [Internet]. Recomendaciones de consenso respecto al soporte respiratorio no invasivo en el paciente adulto con insuficiencia respiratoria aguda secundaria a infección por SARS-CoV-2. Última visita: 22 de marzo de 2020. Disponible en: https:// www.separ.es/node/1754

13. Dhama K, Sharun K, Tiwari R, Dadar M, Malik YS, Singh KP, et al. COVID-19, an emerging coronavirus infection: advances and prospects in designing and developing vaccines, immunotherapeutics, and therapeutics. Hum Vaccin Immunother. 2020;16(6):1232-8. doi: 10.1080/21645515.2020.1735227.

14. World Health Organization [Internet]. Infection prevention and control of epidemic-and pandemic prone acute respiratory infections in health care. Última visita: 22 de marzo de 2020]. Disponible en: https://www.who.int/publications/i/item/infection-prevention-and-control-of-epidemic-and-pandemicprone-acute-respiratory-infections-in-health-care

15. Pittet D, Allegranzi B, Boyce J, World Health Organization World Alliance for Patient Safety First Global Patient Safety Challenge Core Group of Experts. The World Health Organization guidelines on hand hygiene in health care and their consensus recommendations. Infect Control Hosp Epidemiol. 2009;30(7):611-22. doi: 10.1086/600379.

16. Instituto Nacional de Vigilancia de Medicamentos y Alimentos [Internet]. Consec expediente producto registro sanitario vigencia principio activo presentación comercial forma farmacéutica vía de admin. Última visita: 22 de marzo de 2020]. Disponible en: https://www.invima.gov.co/documents/20143/453029/ RS_4_Vacunas_Enero_2018.pdf/1357cc96-4fde-8e50-ca21$3 \mathrm{c} 91 \mathrm{c} 5274 \mathrm{~d} 95 ? \mathrm{t}=1541012269514$

17. Jefferson T, Del Mar CB, Dooley L, Ferroni E, Al-Ansary LA, Bawazeer GA, et al. Physical interventions to interrupt or reduce the spread of respiratory viruses. Cochrane Data- 
base Syst Rev. 2011;(7):CD006207. doi: 10.1002/14651858. CD006207.pub4.

18. Yang CL, Ducharme FM, Radhakrishnan D, Podgers D, Ramsey C, et al. Key highlights from the Canadian Thoracic Society Position Statement on the Optimization of Asthma Management During the COVID-19 Pandemic. Chest. 2020;158(4):1335-7. doi: 10.1016/j.chest.2020.05.551.

19. National Institute for Health and Care Excellence [Internet]. COVID-19 rapid guideline: severe asthma. NICE guideline. Publicado el: 3 de abril de 2020. Disponible en: www.nice. org.uk/guidance/ng166

20. Esquivel A, Busse WW, Calatroni A, Togias AG, Grindle KG, et al. Effects of omalizumab on rhinovirus infections, illnesses, and exacerbations of asthma. Am J Respir Crit Care Med. 2017;196(8):985-92. doi: 10.1164/rccm.201701-0120OC.

21. Shaker MS, Oppenheimer J, Grayson M, Stukus D, Hartog $\mathrm{N}$, et al. COVID-19: pandemic contingency planning for the allergy and immunology clinic. J Allergy Clin Immunol Pract. 2020;8(5):1477-88.e5. doi: 10.1016/j.jaip.2020.03.012.
22. Organización Panamericana de la Salud, Organización Mundial de la Salud [Internet]. Prevención y control de infecciones. Materiales científicos y técnicos. Última visita: 22 de marzo de 2020. Disponible en: https:/www.paho.org/es/ documentos/presentacion-prevencion-control-infeccionesnuevo-coronavirus-covid-19-precauciones

23. Asthma and Respiratory Foundation NZ [Internet]. Coronavirus and children with respiratory conditions. Última visita: 22 de marzo de 2020. Disponible en: https://www.asthmafoundation.org.nz/news-events/2020/coronavirus-and-childrenwith-respiratory-conditions

24. Sociedad Española de Inmunología Clínica, Alergología y Asma Pediátrica [Internet]. Recomendaciones específicas sobre coronavirus para niños asmáticos y alérgicos. Última visita: 22 de marzo de 2020. Disponible en: http://www. seicap.es/es/recomendaciones-espec $\% \mathrm{C} 3 \%$ ADficas-sobrecoronavirus-para-ni $\% \mathrm{C} 3 \% \mathrm{~B} 1$ os-asm $\% \mathrm{C} 3 \% \mathrm{~A} 1$ ticos-yal\%C3\%A9rgicos_82387 